Rienhoff had long been tapping experts such as Dietz for assistance. In 2005, an examination at Johns Hopkins revealed Bea's bifid uvula. This feature, combined with others, suggested Loeys-Dietz syndrome, which is caused by mutations in TGF- $\beta$ receptors. But physicians found none of the known mutations after sequencing these genes individually. This was a relief: Loeys-Dietz is associated with devastating cardiovascular complications and an average life span of 26 years.

In 2008, Jay Flatley, chief executive of Illumina, offered Rienhoff the chance to sequence Bea's transcriptome - all of the RNA expressed by a sample of her cells along with those of her parents and her two brothers. After drilling into the data, Rienhoff and his collaborators found that Bea had inherited from each parent a defectivelooking copy of CPNE1, a poorly studied gene that seems to encode a membrane protein. It looked like the answer.

But questions remained. The gene did not have obvious connections to Bea's features, and publicly available genome data suggests that the CPNE1 mutations are present in about 1 in 1,000 people - an indication that there should be many more people like Bea.

Unsatisfied, Rienhoff went back to Illumina in 2009 to ask for more help. He proposed exome sequencing, which captures the whole protein-encoding portion of the genome, and is in some ways more comprehensive than transcriptome sequencing. At the time, Illumina was developing its exome-sequencing technology, and the company again took on the Rienhoff family as a test group.

The analysis pulled up a mutation in one copy of the gene that encodes TGF- $\beta 3$ — just in Bea. In cell culture and experiments in frog eggs, the faulty gene seems to produce a non-functional protein that reduces TGF- $\beta$ signalling. This mechanism would differ from what many suspect is going on in Marfan and Loeys-Dietz syndromes, in which mutations paradoxically amp up TGF- $\beta$ signalling. A collaborator of Rienhoff is now engineering a mouse that shares Bea's gene variant, which could help to clarify whether the mutation revs up signalling or suppresses it.

The latest study does not define a new 'Rienhoff syndrome'. For that, Rienhoff and his collaborators would need to find other patients who share Bea's features and genetic markers. Rienhoff says that he would be relieved if he found an older person with similar symptoms who seems as vivacious as his daughter, who recently earned an orange belt in karate; it would tell him that cardiovascular complications are not pre-ordained. "If I saw a single case, I might say, 'Hallelujah', he says.

\section{Proof mooted for quantum uncertainty}

\section{BY RON COWEN}

$\mathrm{E}$ ncapsulating the strangeness of quantum mechanics is a single mathematical expression. According to every undergraduate physics textbook, the uncertainty principle states that it is impossible to simultaneously know the exact position and momentum of a subatomic particle - the more precisely one knows the particle's position at a given moment, the less precisely one can know the value of its momentum.

But the original version of the principle, put forward by physicist Werner Heisenberg in 1927, couches quantum indeterminism in a different way - as a fundamental limit to how well a detector can measure quantum properties. Heisenberg offered no direct proof for this version of his principle, and expressed his ideas "only informally and intuitively", says physicist Jos Uffink of the University of Minnesota in Minneapolis.

Now researchers say that they have a formal proof. "Our work shows that you can't measure something with an accuracy any better than the fundamental quantum uncertainty," says Paul Busch, a theoretical physicist at the University of York, UK, who with his colleagues posted the proof on 6 June on the arXiv preprint server ${ }^{1}$. Not only does the work place this measurement aspect of the uncertainty principle on solid ground - something that researchers had started to question - but it also suggests that quantum-encrypted messages can be transmitted securely.

In their theoretical work, Busch and his colleagues imagined making simultaneous measurements of a particle's position and momentum in an arbitrary quantum state. They compared the errors in such measurements to two special cases - in which either the position or the momentum of the particle is well known. They found that the combined errors in measurements of the position or momentum in these two cases obeyed Heisenberg's principle and was always smaller than for cases in which the two properties were measured simultaneously. This step allowed them to prove Heisenberg's original conjecture.

Busch and his co-authors' work "is worth being remembered and maybe even taught through textbooks as the proper version of the Heisenberg error-disturbance relation", says mathematician Hans Maassen of Radboud University in Nijmegen, the Netherlands. However, the proof has reignited a decade-long debate.

\section{POLARIZED DEBATE}

In 2003, physicist Masanao Ozawa, now at Nagoya University in Japan, proposed that the measurement-disturbance version of the Heisenberg uncertainty principle could be violated. Ozawa's theory was published in 2004 (ref. 2) and was corroborated last year, when Aephraim Steinberg and Lee Rozema at the University of Toronto, Canada, and their colleagues reported the results of an experiment in which they measured the polarization of a single photon along two perpendicular directions ${ }^{3}$.

Just like position and momentum, quantum theory predicts that the polarization along two different axes cannot simultaneously be known with certainty (see Nature http://doi. org/m27; 2012). The team adopted a strategy in which the polarization is initially probed using a series of 'weak' measurements - detections that barely disturb the system but must be repeated several times to record the same information that a single 'strong' measurement can detect. They found that, on average, the

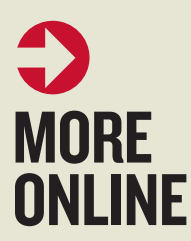

NEWS

- Online games offer trove of brain data go.nature.com/ujowtc - Violence against women at epidemic proportions go.nature. com/xtgmoh

- Blocking boozy memories reduces risk of relapse go.nature. com/wppqan
Whole human brain is mapped in 3D go.nature.com/os/b4s 
> polarization measurements disturbed the system by only about half as much as Heisenberg's original formulation of the uncertainty principle dictates.

Ozawa says that Busch and his collaborators use the worst-case scenario, averaged over many quantum states, to define the disturbance caused by a measuring device. That may not reflect the actual conditions under which a particular quantum system in a particular quantum state is being examined. As a result, Ozawa says, the authors are overestimating measurement errors. But Busch and his colleagues argue that the definition of instrument error Ozawa uses is not universally valid, and therefore does not call into question Heisenberg's principle.

\section{CRYPTIC CLUES}

The debate may sound esoteric, but quantifying by how much a measuring device can disturb the properties of a quantum system is crucial to the burgeoning field of quantum cryptography and computing. In principle, a quantum computer would be more secure than an ordinary computer because anyone trying to peer at the information would disturb it, leaving a telltale trace.

If Ozawa's 2004 work holds up, it would mean that a thief might be able to steal quantum data without anyone knowing, as the furtive measurements might barely disturb the system at all.

But what Busch and his collaborators have proved, says Uffink, is that if an eavesdropper has no control over the state of the quantum system in question - as will typically be the case for a data thief - then the eavesdropper will necessarily disturb the system in the way that the original version of the uncertainty principle predicts. "This is an important result that we did not have before," Uffink says.

Neither Uffink nor quantum theorist Howard Wiseman at Griffith University in Brisbane, Australia, are willing to say that Ozawa's approach is wrong, however. Indeed, it is possible that both results are correct, Wiseman notes.

He suspects that a strange quantum concept known as negative probability - negative dips in the probability distribution of a particle's location or momentum - could be at the heart of the issue. These dips may mean that a measuring device disturbs the system less than the uncertainty principle seems to allow. "The fact these two different definitions give you a different answer is telling you something about the weirdness of quantum mechanics," says Wiseman.

1. Busch, P., Lahti, P. \& Werner, R. F. Preprint available at http://arxiv.org/abs/1306.1565 (2013).

2. Ozawa, M. Ann. Phys. 311, 350-416 (2004).

3. Rozema, L. A. et al. Phys. Rev. Lett. 109, 100404 (2012).

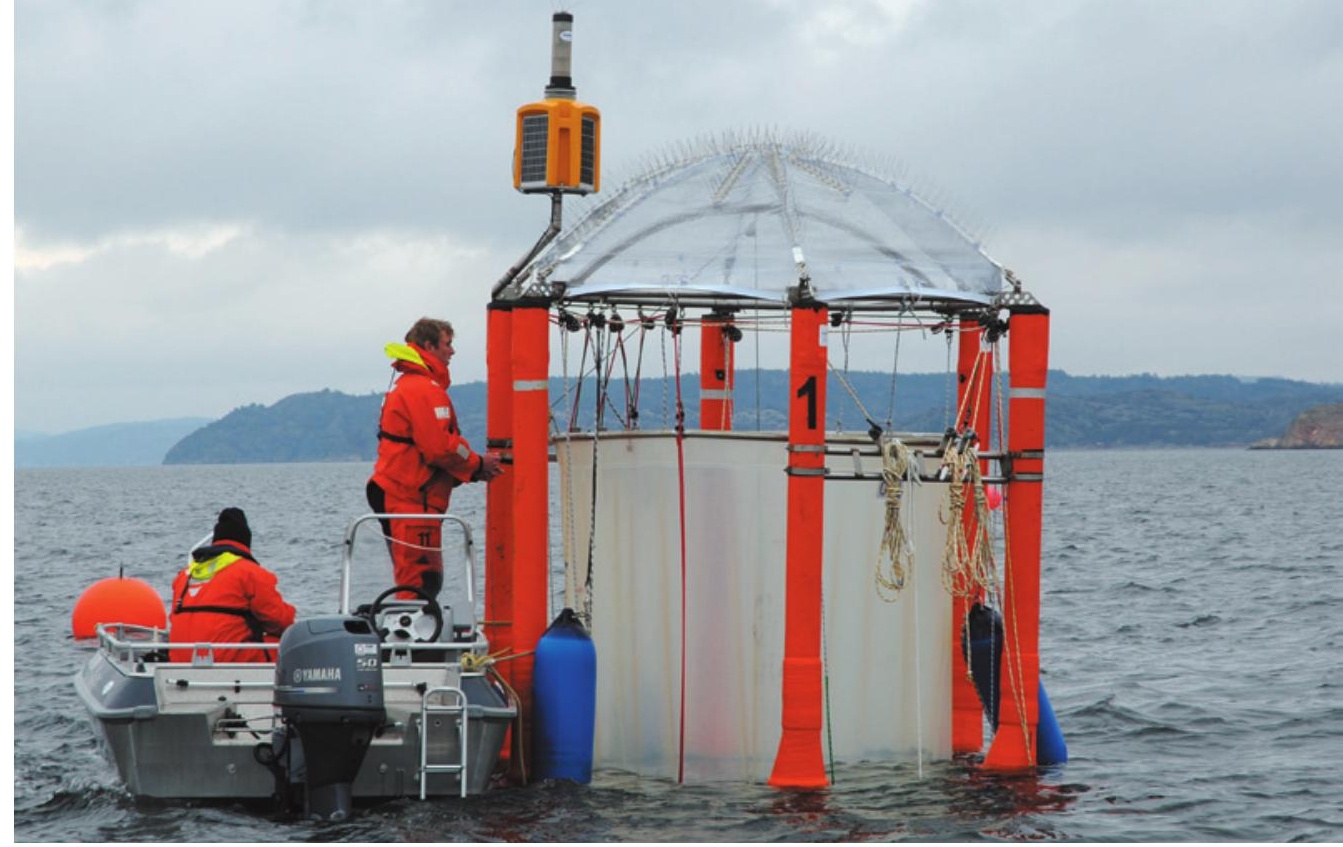

Researchers suspend 20-metre-tall sacs in a Swedish fjord to enclose entire ecosystems for study.

MARINE ECOLOGY

Ocean labs probe effects of ocean acidification on ecosystems.

\section{BY HRISTIO BOYTCHEV}

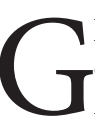
lobal warming is not the only worrying consequence of rising carbon emissions. As levels of carbon dioxide increase in the atmosphere, more of the gas dissolves into the oceans, making the water more acidic. Marine scientists fear that the conditions will disrupt ecosystems by, for example, inhibiting some organisms' ability to build shells. Yet the effects are unclear: in small-scale laboratory tests, certain species have proved surprisingly resilient, and some even flourish.

Marine biologist Ulf Riebesell says that these results tell only part of the story: scientists need to scale up and examine whole ecosystems. Lab studies of isolated species ignore variables such as competition, predation and disease, he says. Even minor effects of acidification on the fitness of individual species - especially small photosynthetic organisms such as phytoplankton - can upset food chains, eventually harming larger species. "If you only focus on the lab results, you are being misled," he says.

Riebesell and his colleagues at GEOMAR Helmholtz Centre for Ocean Research in Kiel, Germany, have developed innovative experimental environments - 20-metre-tall sacs suspended in the ocean, which enclose entire ecosystems and allow the effects of elevated $\mathrm{CO}_{2}$ to be measured. The first results, published this year, suggest that some plankton thrive in acidic environments and can wreak havoc on food chains ${ }^{1}$. Another experiment will end in July, and preliminary evidence suggests that conches and sea urchins are vulnerable to acidification.

The project is inspired by analogues on land, in which swathes of forest are bathed in extra $\mathrm{CO}_{2}$ to study the effects on plant life (see Nature 496, 405-406; 2013). For the sea, Riebesell and his colleagues constructed 'mesocosms' - floating cylinders of thin plastic that function like giant test tubes ${ }^{2}$. When first put into the water, the sacs are left open at the top and bottom, allowing hundreds of small species to enter. After several days, they are closed and acidified water is pumped in (see 'Sea lab'). Over weeks or months, researchers measure how the ecosystems inside fare in comparison with those in untreated sacs.

Realizing this simple idea has been challenging. The scientists began in 2006 with a prototype, free-floating in the Baltic Sea, that floated too well: currents carried it along much faster than expected, and the scientists had to chase it in a research ship. After only two days they reached Swedish waters, for which they had no research permits. When they tried to recover the mesocosm, it broke.

The team conducted its first successful experiment in 2010, using a lighter design that $\rightarrow$ NATURE.COM

For more on ocean acidification, see: go.nature.com/ihz4qy was moored in place in the Norwegian Arctic archipelago of Svalbard. The researchers found that, compared with the 